\section{Reflective interferometric chamber for quantitative phase imaging of biological sample dynamics}

\author{
Natan T. Shaked, ${ }^{*}$ Yizheng Zhu, Nima Badie, \\ Nenad Bursac, and Adam Wax \\ Duke University, Department of Biomedical Engineering, \\ Fitzpatrick Institute for Photonics, Durham, \\ North Carolina 27708
}

\begin{abstract}
We introduce a new interferometric setup for single-exposure wide-field holographic phase imaging of highly dynamic biological samples. In this setup, the interferometric signal originates from a specially designed reflective interferometric chamber (InCh), creating an off-axis interferogram on the output plane of the system. The setup only requires the $\mathrm{InCh}$ and a simple reflectionmode two lens imaging system, without the need for additional optical elements such as gratings in the beam path. In addition, due to the close-to-common-path geometry of the setup, phase noise is greatly reduced. We experimentally compare the inherent phase stability of the system in ambient conditions to that of a conventional interferometer. We also demonstrate use of this system for wide-field quantitative phase imaging of two different highly dynamic, optically transparent biological samples: beating myocardial cells and moving unicellular microorganisms. $\odot 2010$ Society of Photo-Optical Instrumentation Engineers. [DOI: 10.1117/1.3420179]
\end{abstract}

Keywords: phase microscopy; cell imaging; myocardial cells; myocytes; euglena.

Paper 10098LR received Feb. 25, 2010; revised manuscript received Mar. 28, 2010; accepted for publication Apr. 1, 2010; published online Jun. 3, 2010.

Optically transparent biological samples, such as isolated biological cells and certain microorganisms in culture, are 3-D dynamic entities, constantly adjusting their biophysical features, shapes, volumes, and spatial locations. These intrinsic transients can occur over time scales ranging from days to less than milliseconds. Tracking these dynamic phenomena can provide valuable spatial-temporal data for cell biology studies, as well as provide early indications of malfunctions due to disease, which can be used as biomarkers. Wide-field digital interferometry (WFDI) is a label-free technique providing quantitative measurements of optical path delays (OPDs) associated with optically transparent samples. ${ }^{1-4}$ By recording the interference pattern between the light that has interacted with the sample and the mutually coherent reference wave, WFDI captures the complex wavefront of the sample field, containing the quantitative phase profile of the sample.

WFDI phase measurements of transparent biological sample dynamics are frequently performed by modified Mach-Zehnder or Michelson interferometric setups, where at least one microscope objective is inserted in the beam path to

*Tel: 919-660-5588: Fax: 919-684-4488; E-mail: natan.shaked@duke.edu

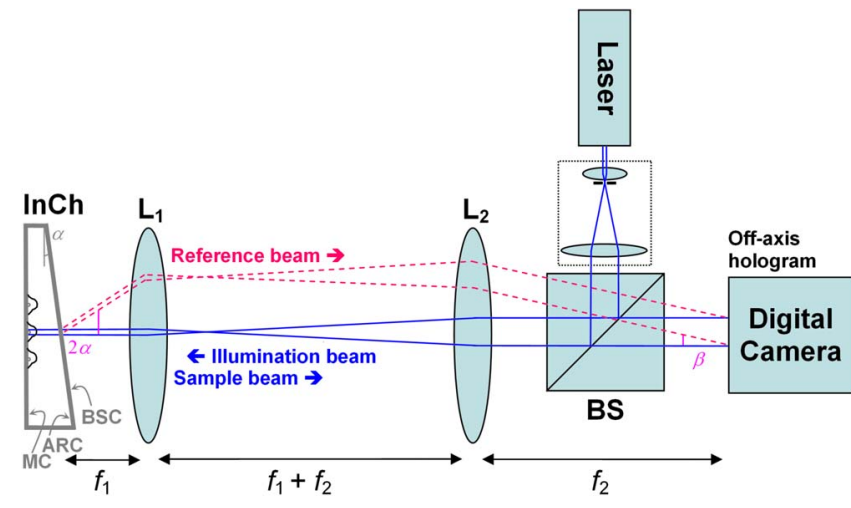

Fig. 1 InCh setup for single-exposure holographic phase imaging of biological samples. $\mathrm{L}_{1}, \mathrm{~L}_{2}=$ lenses, $\mathrm{SPF}=$ spatial filter/beam expander (two lenses and a confocally positioned pinhole), BS=beamsplitter, $M C=$ mirror coating (on the front face of the rear coverslip), ARC = antireflection coating (on the rear face of the front coverslip), and $\mathrm{BSC}=$ beamsplitter coating (on the front face of the front coverslip).

magnify the sample. The reference and sample arms in these conventional interferometers have different optical paths. The introduction of any kind of instability in the interferometric system, including differential vibrations or air perturbations in the interferometer arms, causes measurement errors and can significantly degrade the quality of the recorded phase profile of the sample.

Common-path interferometers provide a partial solution to the instability problem. This is achieved by creating an overlap between the reference and sample beams, so that the same vibrations occur for both beam paths. Common-path interferometers are particularly useful in ambient conditions, where the interferometric system is not constructed on vibrationisolating optical tables or the system cannot be enclosed to avoid differential air perturbations in the two interferometer arms.

Common-path interferometry can be implemented using WFDI on-axis geometry, ${ }^{5}$ requiring the acquisition of several phase-shifted interferograms for each instance of the sample, or using off-axis geometry, ${ }^{4,6}$ requiring the acquisition of only one interferogram per sample instance. The latter approach, however, requires the use of a diffraction grating and often other elements in the beam paths, which necessitates a dedicated optical system design and an additional alignment.

This work introduces the interferometric chamber ( $\mathrm{InCh}$ ), as an alterative approach for off-axis close-to-common-path interferometers for recording the dynamics of transparent biological samples. In this design, splitting the beams at the desired angle is performed by the chamber itself, where no special optical elements are required in the beam path. By integrating the interferometric elements of the optical system into the InCh (a single rigid device), the interferometric alignment can be performed once, during the fabrication of the chamber and not each time before the measurement.

Figure 1 presents the suggested close-to-common-path interferometer. As can be seen in this figure, coherent light from a laser source is spatially filtered and expanded. The area of the generated plane wave should be larger than the area of the recorded field of view on the digital camera. This plane wave 
is first reflected by a beamsplitter and then passes through a $4 \mathrm{f}$ optical system, containing two lenses $\mathrm{L}_{1}$ and $\mathrm{L}_{2}$ with focal lengths of $f_{1}$ and $f_{2}$, respectively, which demagnify the illumination beam before reaching the InCh. The InCh consists of two coverslips. The front coverslip of the $\mathrm{InCh}$ is inclined at an angle $\alpha$, whereas the rear coverslip is normal to the optical axis. When hitting the front coverslip, part of the incident beam is reflected at an angle of $2 \alpha$ relative to the optical axis. This reflected beam serves as the reference beam. To ensure that lens $\mathrm{L}_{1}$ will collect the oblique reference beam, its numerical aperture (NA) should be at least $n \sin (2 \alpha)$, where $n$ is the refractive index of the immersion medium between lens $\mathrm{L}_{1}$ and the sample. The beam transmitted through the front coverslip is reflected from the rear coverslip of the InCh and leaves the chamber after interacting with the transparent sample twice. This beam is the sample beam. The sample and reference beams are projected by the two lenses onto the camera, while the sample beam propagates along the optical axis and the reference beam reaches the digital camera at an incidence angle of $\beta=\arctan \left[\tan (2 \alpha) \cdot f_{1} / f_{2}\right]$. This angle must be adjusted so that the carrier frequency of the interferogram will not exceed the Nyquist frequency of the camera. This will happen if $\beta \leqslant \beta_{\max }=\arcsin [\lambda /(2 \Delta)]$, where $\lambda$ is the wavelength of the source and $\Delta$ is the pixel size of the digital camera. For example, if $\Delta=7.4 \mu \mathrm{m}$ and $\lambda=633 \mathrm{~nm}$, we get $\beta_{\max }=2.45 \mathrm{deg}$. If we choose $\mathrm{L}_{1}$ and $\mathrm{L}_{2}$ that yield a magnification of $f_{2} / f_{1}=10$, the InCh's front coverslip inclination angle should satisfy $\alpha \leqslant \arctan \left[\tan \left(\beta_{\max }\right) \cdot f_{2} / f_{1}\right] / 2$ $=11.58 \mathrm{deg}$. If $\alpha=11.58 \mathrm{deg}$ is chosen (so that $\beta=\beta_{\max }$ ), and assuming that the immersion medium is air, $\mathrm{L}_{1}$ should have a NA of $\sin (2 \alpha) \cong 0.39$ or larger to ensure collection of the inclined reference beam by $\mathrm{L}_{1}$. The hologram captured by the digital camera is then digitally processed to yield the unwrapped phase profile of the sample by the off-axis WFDI digital process explained in our previous work, ${ }^{4}$ where for the InCh, the measured phase is twice the sample phase. Note that since the sample beam passes through the sample twice, the system will more accurately measure phase profiles of transparent samples that are flatly attached to the rear coverslip of the InCh. This condition is generally valid for cell cultures after several hours of incubation.

In the demonstrations presented here, the spatial resolution of the phase profile is limited by the detector pixel size and not by the NA of the imaging optics. Thus, the inclination angle of the front coverslip of the InCh is determined by the camera pixel size, as explained above. However, in the case of a diffraction-limited system, the spatial resolution of the phase profile will be limited by the angle of the reference beam, rather than by the full NA of the optics.

The InCh can be implemented using two simple coverslips. However, in this case most of the illuminating light will be lost while transmitted through the chamber, rather than reflected to the camera. In addition, inner reflections from the unused sides of the coverslips will partially occlude the hologram. Therefore, to create a 1:1 ratio between the reference and sample beam intensities, the front face of the rear coverslip should be coated by a mirror coating (100\% reflection), and the front face of the front coverslip by $38 \%$ reflection coating, for forming the reflected reference beam. Since the sample beam passes through the front coverslip twice, it will also have $62 \times 62 \%=38 \%$ of the initial intensity of the illu-

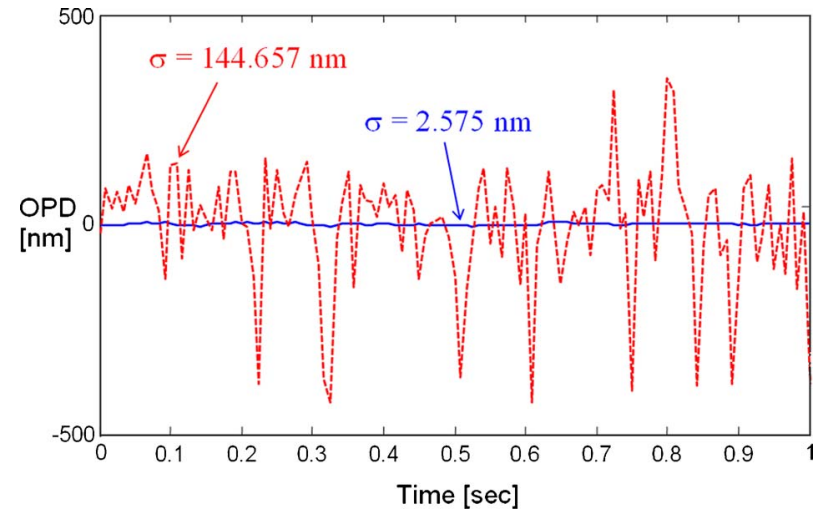

Fig. 2 Inherent temporal OPD stability of two interferometric systems in ambient conditions (nonfloating optical table, no enclosure) at 120 frames per second: the InCh-based system (solid line), and a Michelson interferometer (dashed line). Both systems are built on the same table and with the same equipment.

mination beam. In addition, antireflection coating can be used in the rear face of the front coverslip to avoid unwanted reflections. For the experiments performed in this work, we have chosen to coat the rear coverslip with a 45-nm layer of gold (3\% transmission measured), forming a mirror, and the front face of the front coverslip with a 7-nm layer of gold (63\% transmission measured), forming a beamsplitter. We used a $17-\mathrm{mW}$ HeNe source, lenses with NAs of 0.45 that formed $f_{2} / f_{1}=10 \times$ magnification, and an AVT Pike 032-B digital camera (Allied Vision Technologies, Stadtroda, Germany).

To illustrate the utility of the proposed system as a closeto-common-path interferometer, we have first examined the inherent stability of the phase measurement in ambient conditions, where the optical table does not float and the system is not caged to avoid regular-room air perturbations. This stability characterization was first performed for the InCh-based system shown in Fig. 1, which results in OPD fluctuations with a standard deviation of $2.575 \mathrm{~nm}$ (solid-line graph shown in Fig. 2), an excellent stability for these ambient conditions. For comparison, we have used the same system to implement a conventional Michelson interferometer. This is performed by removing the front (inclined) coverslip of the InCh and positioning a mirror after the unused face of the beamsplitter. Under the same ambient conditions, this conventional Michelson interferometer has yielded OPD fluctuations with a standard deviation of $144.697 \mathrm{~nm}$ (dashed-line graph in Fig. 2), more than 1.5 orders of magnitude worse than that obtained by the InCh-based system. To illustrate the system's inherent stability in tightly controlled laboratory conditions, the experiment was repeated with a floating optical table and a system enclosure to prevent ambient air vibrations, resulting in OPD fluctuations with a standard deviation of $0.2 \mathrm{~nm}$. This result is comparable or better than the results obtained by other off-axis close-to-common-path interferometers (e.g., Ref. 2), but without the use of specialized optical elements in the beam path.

Next, we have used the InCh-based system to acquire phase profiles of two different highly dynamic transparent biological samples: beating myocardial cells in culture and an Euglena gracilis, a unicellular protist, moving in spring water. 


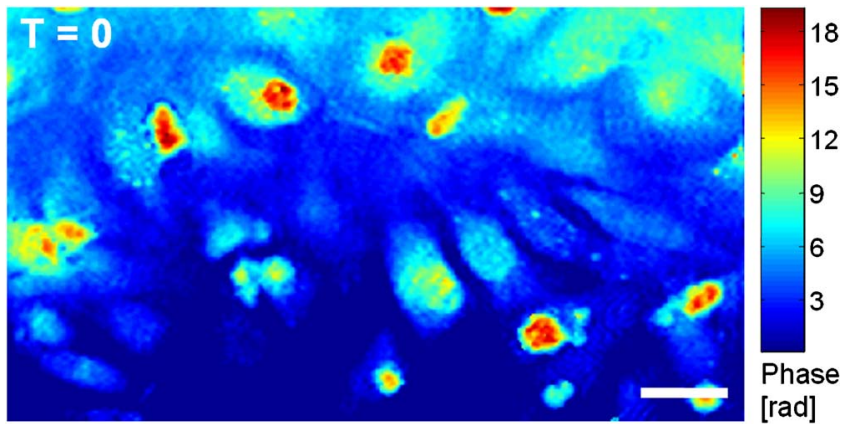

Video 1 InCh-based WFDI phase imaging of beating rat myocardial cells in vitro. White scale bar represents $40 \mu \mathrm{m}$ (QuickTime, 1.09 MB). [URL: http://dx.doi.org/10.1117/1.3420179.1].

For the myocardial cell sample preparation, ventricular cardiomyocytes were enzymatically isolated from 2-day-old neonatal Sprague-Dawley rats as previously described. ${ }^{7}$ The cells were diluted in cell seeding medium to a concentration of 120,000 cells $/ \mathrm{mL}$. The mirror-coated (rear) coverslip of the InCh was recoated with fibronectin $[25 \mu \mathrm{g} / \mathrm{mL}$ in phosphate buffered saline (PBS)] by placing it face down on a $200-\mu \mathrm{L}$ drop for $1 \mathrm{~h}$ at room temperature. ${ }^{7}$ Each coverslip was seeded with $2 \mathrm{~mL}$ of the cell suspension and incubated at $37^{\circ} \mathrm{C}$ for 2 days, when cells were completely adhered to the coverslip. Prior to the measurement, epinephrine $(2 \mathrm{mM})$ was added to the suspension to enhance cell contractility.

Video 1 presents the spatial-temporal quantitative phase profiles of the myocardial cell culture obtained by the InChbased system. As shown, individual cells continuously change their 3-D phase profiles rapidly and beat at different rates. As will be shown in our future work, the technique is useful for examining various biophysical properties of myocardial cells in culture.

The second dynamic sample, which was chosen to demonstrate the quantitative phase acquisition capabilities of the proposed system, contained Euglena gracilis from a bacteriaenhanced culture (obtained from Carolina Biological Supply, Burlington, North Carolina). During the measurement, the Euglenas were forced to swim close to the rear, mirror-coated coverslip of the InCh by attaching another coverslip to it. This pressure application caused some euglenas (e.g., the lower euglena in Video 2) to swim relatively slower than usual. On the other hand, some euglenas (e.g., the upper euglena in Video 2) changed their 3-D shapes fast due to the high pressure exerted on them ${ }^{8}$ by the additional coverslip.

To conclude, we propose a new close-to-common-path single-exposure WFDI approach for obtaining quantitative phase profiles of transparent biological samples. The new approach is based on the design of a specialized interferometric chamber, the $\mathrm{InCh}$, and a simple two-lens system. Compared to a conventional Michelson interferometric setup, this system provides more than 1.5 orders of magnitude improvement of phase stability in ambient conditions. We also demonstrate use of the InCh system with two highly dynamic, optically transparent biological samples. We believe that the proposed close-to-common-path design can be an important tool for high-precision WFDI acquisition of the phase profiles of biomedical samples in configurations that do not allow for the

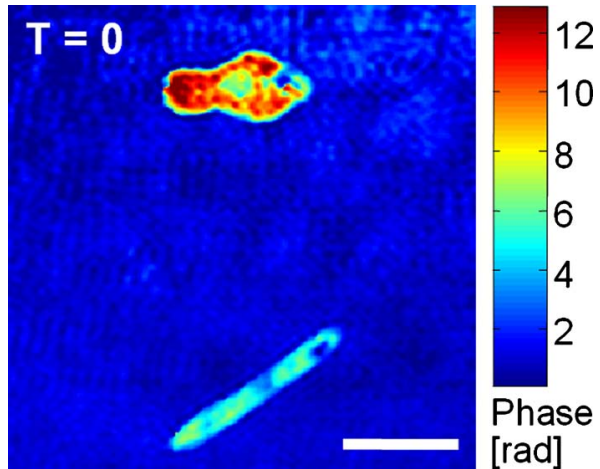

Video 2 InCh-based WFDI phase imaging of Euglena gracilis (unicellular microorganism) under pressure. White scale bar represents $40 \mu \mathrm{m}$ (QuickTime, 395 KB).

[URL: http://dx.doi.org/10.1117/1.3420179.2].

strict stability conditions that are usually needed for WFDI acquisition. In addition, the fact that the entire interferometric signal originates from the chamber itself adds further simplicity to the design, enabling the end user to record holograms of biological samples without building the interferometric system and aligning it. A different InCh geometry should be used when the magnification is changed within a given optical system. However, due to the InCh simple design, only the inclination angle of the front coverslip has to be changed. We thus estimate that the cost of the chamber fabrication in a mass production can be as little as a few dollars per unit. Additionally, considering the fact that only the InCh and a simple two-lens system are required in this setup, the InCh might be useful in regular reflection-mode microscopes.

\section{Acknowledgments}

Supported by NSF grant CBET-0651622. We thank Joseph Blum from the Department of Cell Biology at Duke University for his useful advice regarding the Euglena gracilis experiment. Shaked greatly acknowledges the support of the Bikura Postdoctoral Fellowship from Israel.

\section{References}

1. B. Rappaz, P. Marquet, E. Cuche, Y. Emery, C. Depeursinge, and P. J. Magistretti, "Measurement of the integral refractive index and dynamic cell morphometry of living cells with digital holographic microscopy," Opt. Express 13, 9361-9373 (2005).

2. G. Popescu, T. Ikeda, R. R. Dasari, and M. S. Feld, "Diffraction phase microscopy for quantifying cell structure and dynamics," Opt. Lett. 31, 775-777 (2006).

3. N. T. Shaked, M. T. Rinehart, and A. Wax, "Dual-interferencechannel quantitative-phase microscopy of live cell dynamics," Opt. Lett. 34, 767-769 (2009).

4. N. T. Shaked, J. D. Finan, F. Guilak, and A. Wax, "Quantitative phase microscopy of articular chondrocyte dynamics by wide-field digital interferometry," J. Biomed. Opt. Lett. 15, 010505 (2010).

5. C. S. Anderson, "Fringe visibility, irradiance, and accuracy in common path interferometers for visualization of phase disturbances," Appl. Opt. 34, 7474-7485 (1995).

6. V. Micó, Z. Zalevsky, and J. García, "Superresolution optical system by common-path interferometry," Opt. Express 14, 5168-5177 (2006).

7. N. Badie and N. Bursac, "Novel micropatterned cardiac cell cultures with realistic ventricular microstructure," Biophys. J. 96, 3873-3885 (2009).

8. J. Byrne and D. Marsland, "Pressure-temperature effects on the formstability and movements of Euglena gracilis var. Z," J. Cell. Comp. Physiol. 65, 277-284 (1965). 